\title{
A follow-up of integrated positron emission tomography/computed tomography after curative resection of non-small-cell lung cancer in asymptomatic patients
}

\author{
Sukki Cho, MD, and Eung Bae Lee, MD, PhD
}

\begin{abstract}
Objective: A follow-up integrated positron emission tomography/computed tomography (PET/CT), as part of a more intensive surveillance program, has been performed at around 1 year after curative resection, regardless of the patients' symptoms or findings in other tests. This study was designed to evaluate the results of this followup integrated PET/CT in patients treated for non-small-cell lung cancer without symptoms or abnormal findings.
\end{abstract}

Methods: Between January 2003 and December 2006, this study enrolled 86 patients with non-small cell lung cancer who had no clinical or radiologic evidence of recurrence after curative resection before integrated PET/CT and underwent a follow-up integrated PET/CT around 1 year at our institution.

Results: The time from operation to the follow-up integrated PET/CT check was $13.4 \pm 4.4$ months. Integrated $\mathrm{PET} / \mathrm{CT}$ showed negative findings in $41(47.7 \%)$ patients, equivocal findings in $16(18.6 \%)$ patients, and positive findings in $29(33.7 \%)$ patients. Twenty-seven $(31.4 \%)$ patients had recurrent disease and 2 patients had extrathoracic double primary cancer. Six patients had extrathoracic recurrence without intrathoracic recurrence.

Conclusions: A postoperative follow-up integrated PET/CT can be used for early detection of recurrence in asymptomatic patients who had had resection of non-small-cell lung cancer. Further studies are required to evaluate the cost-effectiveness or survival benefit of follow-up integrated PET/CT. (J Thorac Cardiovasc Surg 2010;139:1447-51)

Surgery is the most appropriate treatment for early-stage non-small-cell lung cancer (NSCLC). Although 5-year survivals of more than $60 \%$ can be achieved in selected patients, overall, more than $50 \%$ of those undergoing resection will die of a recurrence. ${ }^{1}$ The majority of recurrences are distant, and more than $80 \%$ occur within the first 2 years. ${ }^{2}$ Many clinicians have tried to detect early signs of second primary lung cancer or recurrence at a treatable stage through postoperative surveillance to increase survival. However, there is no consensus on the best follow-up program after curative resection for NSCLC. Retrospective studies endorsed the concept of less intensive surveillance (symptom-based) because more intensive surveillance has not yet demonstrated benefit in survival and quality of life. ${ }^{3-6}$ In contrast, recent studies have demonstrated that asymptomatic recurrences detected by intensive surveillance had a better prognosis than symptomatic recurrence. ${ }^{2,7}$ As a result, recent guidelines from the National Comprehensive Cancer Network ( $\mathrm{NCCN}$ ) and American College of Chest Physicians (ACCP) recommend

\footnotetext{
From the Department of Thoracic and Cardiovascular Surgery, Kyungpook National University, College of Medicine, Daegu, Korea.

Disclosures: None.

Received for publication April 19, 2009; revisions received Sept 16, 2009; accepted for publication Sept 29, 2009; available ahead of print Dec 14, 2009.

Address for reprints: Sukki Cho, MD, Department of Thoracic and Cardiovascular

Surgery, Kyungpook National University Hospital, 200 Dongduk-Ro, Jung-gu,

Daegu, 700-721, Korea (E-mail: skcho@knu.ac.kr).

$0022-5223 / \$ 36.00$

Copyright $(c) 2010$ by The American Association for Thoracic Surgery

doi:10.1016/j.jtcvs.2009.09.055
}

chest computed tomography (CT) every 6 months for 2 years for surveillance imaging. ${ }^{8,9}$

Although a chest CT can detect changes consistent with recurrence earlier than a chest radiograph, unfortunately the performance characteristics of the chest $\mathrm{CT}$ result in difficulty in distinguishing nonspecific posttreatment changes related to surgery from a recurrence or metachronous double primary lung cancer. In contrast, positron emission tomography (PET) and CT, using ${ }^{18} \mathrm{~F}$-fluorodeoxyglucose (integrated FDG-PET/CT) as a metabolic imaging tool, may be able to distinguish recurrence from scarring, pleural thickening, and mediastinal fibrosis commonly found after surgical resection. ${ }^{10}$ In addition, integrated PET/CT can better assess the status of disease and stratify prognosis than conventional staging and might also affect patient management. $^{11,12}$ Therefore, a follow-up integrated PET/CT, as part of a more intensive surveillance program, has been performed at around 1 year after curative resection in patients without symptoms or abnormal findings in other tests. This study was designed to evaluate the results of this follow-up integrated PET/CT in patients who had NSCLC without symptoms or abnormal findings.

\section{PATIENTS AND METHODS \\ Patients}

This study is a retrospective review of a prospective lung cancer database. We enrolled patients with NSCLC who had no clinical or radiologic evidence of recurrence after curative resection before integrated PET/CT and underwent a follow-up integrated PET/CT around 1 year at our institution. All patients underwent complete anatomic resection and mediastinal 


\section{Abbreviations and Acronyms \\ ACCP = American College of Chest Physicians \\ $\mathrm{CT}=$ computed tomography \\ FDG $={ }^{18}$ F-fluorodeoxyglucose \\ MRI = magnetic resonance imaging \\ NCCN $=$ National Comprehensive Cancer \\ Network \\ NSCLC $=$ non-small-cell lung cancer \\ PET = positron emission tomography}

node dissection. Patients were excluded if they had integrated PET/CT when recurrence was suspected on the basis of symptoms or results of other tests or if they refused PET/CT. Informed consent was obtained from eligible patients after we provided detailed explanations about cost, necessity, and expected effects of integrated PET/CT. This study was approved by the Institutional Review Board of Kyunpook National University Hospital.

\section{Integrated PET/CT Acquisition and Processing}

FDG-PET scans were obtained on a Hi-Rez Reveal PET/CT scanner (CTI Molecular Imaging, Inc, Knoxville, Tenn), which combined a lutetium oxyorthosilicate crystal PET scanner with a 6-slice CT scanner. After fasting for at least 6 hours, patients received an intravenous injection of 370 $\mathrm{MBq}(10 \mathrm{mCi})$ of ${ }^{18} \mathrm{~F}-\mathrm{FDG}$ and were instructed to rest in a quiet room with dimmed lights.

\section{Interpretation of Integrated PET/CT}

$\mathrm{PET} / \mathrm{CT}$ findings were classified as negative if imaging showed no focal FDG uptake suggestive of recurrence. Findings that could not be clearly characterized and that would require follow-up were defined as equivocal. Focal FDG uptake with an intensity higher than that of surrounding tissues was defined as positive.

\section{Postoperative Surveillance}

Follow-ups were conducted every 3 months for 2 years. These included history-taking, chest radiograph, physical examination, and tumor marker (carcinoembryonic antigen, neuron-specific enolase, and/or Cyfra 21-1 but only if elevated before the operation). In addition, a chest $\mathrm{CT}$ was taken every 6 months. If a recurrence was suspected, either through newly presenting symptoms or scheduled tests, then integrated PET/CT was performed. If patients showed no symptoms or abnormal findings in the scheduled test, integrated PET/CT was performed approximately 1 year after curative resection.

\section{Recurrences}

In principle, final diagnosis of recurrence was confirmed by histopathologic examination of samples obtained from surgery or biopsy. If it was impossible to diagnose the recurrence histopathologically, exclusion of recurrent malignancy was based on a clinical and radiologic follow-up period of at least 12 months with no evidence of active malignancy. If brain metastases were suspected in integrated PET/CT, immediate brain magnetic resonance imaging (MRI) was performed in all patients.

\section{RESULTS}

\section{Patient Background}

Eighty-six patients (64 men, 22 women; mean age, 61.2 years) who had no clinical or radiologic evidence of recurrence underwent a follow-up integrated PET/CT to evaluate recurrence around 1 year after curative resection (Table 1).
TABLE 1. Patient characteristics

\begin{tabular}{lcc}
\hline & No. & $\%$ \\
\hline Age (y, mean, range) & 61.2 & $35-76$ \\
Sex, male & 64 & 74.4 \\
Histologic type & & \\
$\quad$ Squamous & 49 & 57.0 \\
Adenocarcinoma & 31 & 36.0 \\
Bronchioloalveolar & 4 & 4.7 \\
Others & 2 & 2.3 \\
Pathologic stage & & \\
IA & 20 & 23.3 \\
IB & 36 & 41.9 \\
IIA & 3 & 3.5 \\
IIB & 12 & 13.9 \\
IIIA & 15 & 17.4 \\
Adjuvant therapy & & \\
Chemotherapy & 8 & 9.3 \\
Radiotherapy & 9 & 2.3 \\
Chemoradiotherapy & 2 & \\
\hline
\end{tabular}

The pathologic staging of the tumors, determined according to the guidelines of the American Joint Cancer Committee, was as follows: 20 patients had stage IA, 36 patients had stage IB, 3 patients had stage IIA, 12 patients had stage IIB, and 15 patients had stage IIIA. The histologic type of the lung cancers, determined according to the World Health Organization classifications, was as follows: $49(57.0 \%)$ cases were squamous cell carcinomas, $31(36.0 \%)$ were adenocarcinomas, and $4(4.7 \%)$ were bronchioloalveolar carcinomas. All patients in the study underwent potentially curative surgery consisting of a lobectomy $(n=83)$ or a pneumonectomy (n $=3$ ) with complete mediastinal lymph node dissection. Twelve patients had neoadjuvant therapy before curative resection. Adjuvant chemotherapy, radiation therapy, or chemoradiotherapy was performed in $8(9.3 \%)$ patients, 9 $(10.5 \%)$ patients, and $2(2.3 \%)$ patients, respectively.

\section{Results of Integrated PET/CT}

The time from operation to the follow-up integrated PET/ CT check was $13.4 \pm 4.4$ months. Results of the integrated PET/CT showed negative findings in $41(47.7 \%)$ patients, equivocal findings in $16(18.6 \%)$ patients, and positive findings in $29(33.7 \%)$ patients (Figure 1).

\section{Recurrence}

Of 86 patients, 27 (31.4\%) patients had recurrent disease with a mean follow-up duration of $31.3 \pm 12.8$ months and 2 $(2.3 \%)$ patients had extrathoracic double primary cancer (Table 2). Recurrence was diagnosed by histologic examination in $9(33.3 \%)$ patients and by serial imaging in 18 $(66.7 \%)$ patients. The most commonly involved organ was the contralateral lung and lymph node in $9(33.3 \%)$ patients, followed by the ipsilateral lung and lymph node in 7 $(25.9 \%)$ patients, the liver in $5(18.5 \%)$ patients, the bone in 

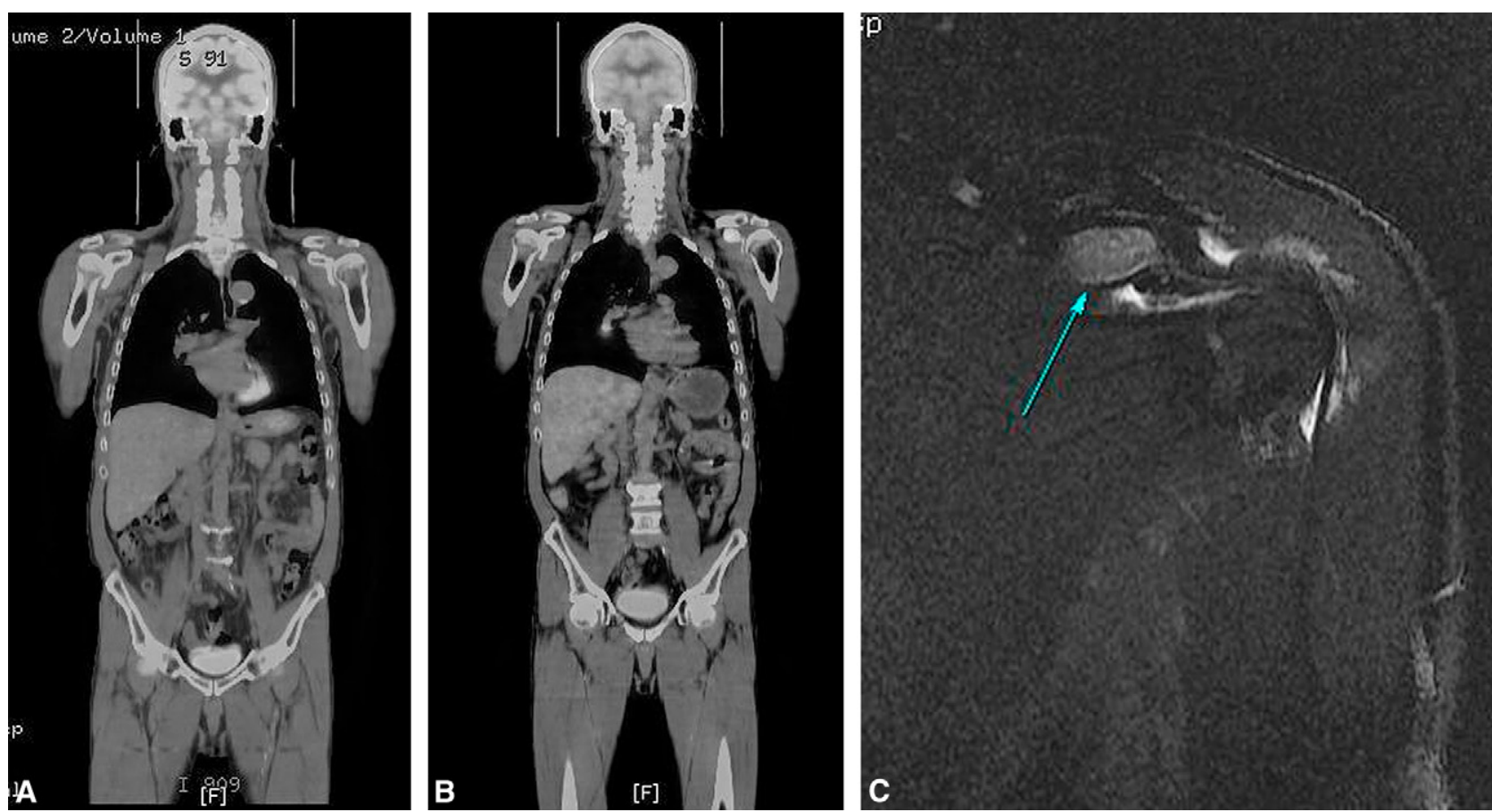

FIGURE 1. One patient had definite evidence of recurrence, which showed newly developed high and focal hypermetabolic lesions. A, A preoperative integrated PET/CT showed no hypermetabolic lesions except for the primary mass. B, A follow-up integrated PET/CT after curative resection showed hypermetabolism at the right interlobar nodes and left scapula, but there were no symptoms related to these lesions. C, Left scapular lesion was confirmed as metastasis of lung cancer by shoulder MRI.

$4(14.8 \%)$ patients, the brain in $3(11.1 \%)$ patients, and the adrenal gland in $2(7.4 \%)$ patients. According to pathologic stage, $9(33.3 \%)$ patients in stage I, $10(37.1 \%)$ patients in stage II, and $8(29.6 \%)$ patients in stage IIIA had a recurrence. Of 16 patients who had a recurrence in lung parenchyma, 9 patients underwent invasive biopsy for histopathologic examination and showed the same histologic type as was present before the first operation. Six $(22.2 \%)$ patients had only extrathoracic distant metastasis

\section{TABLE 2. Recurrence characteristics}

\begin{tabular}{lcc}
\hline & No. & $\%$ \\
\hline No. of recurrences (\% of total patients) & 27 & 31.4 \\
Follow-up duration (mean \pm SD) & $31.3 \pm 12.8$ & \\
Methods by diagnosis & & \\
$\quad$ Histologic examination & 9 & 33.3 \\
$\quad$ Serial follow-up & 18 & 66.7 \\
Locations & & \\
$\quad$ Contralateral lung and lympe node & 9 & 33.3 \\
Ipsilateral lung and lymph node & 7 & 25.9 \\
Liver & 5 & 18.5 \\
Bone & 4 & 14.8 \\
Brain & 3 & 11.1 \\
Adrenal & 2 & 7.4 \\
According to pathologic stage & & \\
I & 9 & 33.3 \\
II & 10 & 37.1 \\
III & 8 & 29.6 \\
\hline
\end{tabular}

without intrathoracic recurrence (Figure 2). Extrathoracic double primary cancer was detected in 2 patients; one was bladder cancer and the other was colon cancer. Both patients underwent surgical resection in the departments of urology and general surgery, respectively.

\section{Postrecurrence Therapy}

Surgery was performed on 4 patients for the initial recurrence. Chest wall resection with wedge resection was performed on 2 patients, only wedge resection on 1 patient, and adrenalectomy on 1 patient. However, 23 patients received nonsurgical therapy: 12 were treated with chemotherapy, 4 with radiotherapy, and 5 with chemoradiation therapy; the remaining 2 patients received supportive care only.

\section{Survival}

Of 27 patients with recurrence, $15(55.6 \%)$ died of disease progression and $12(44.4 \%)$ are still alive with or without recurrence. One patient who underwent adrenalectomy at 14 months after the first operation was still alive at 37 months with no evidence of recurrent disease. Two patients who had extrathoracic double primary cancer are still alive without recurrent lung cancer.

\section{DISCUSSION}

This study showed that integrated PET/CT detected occult metastases in 27 of 86 patients with no symptoms or no 


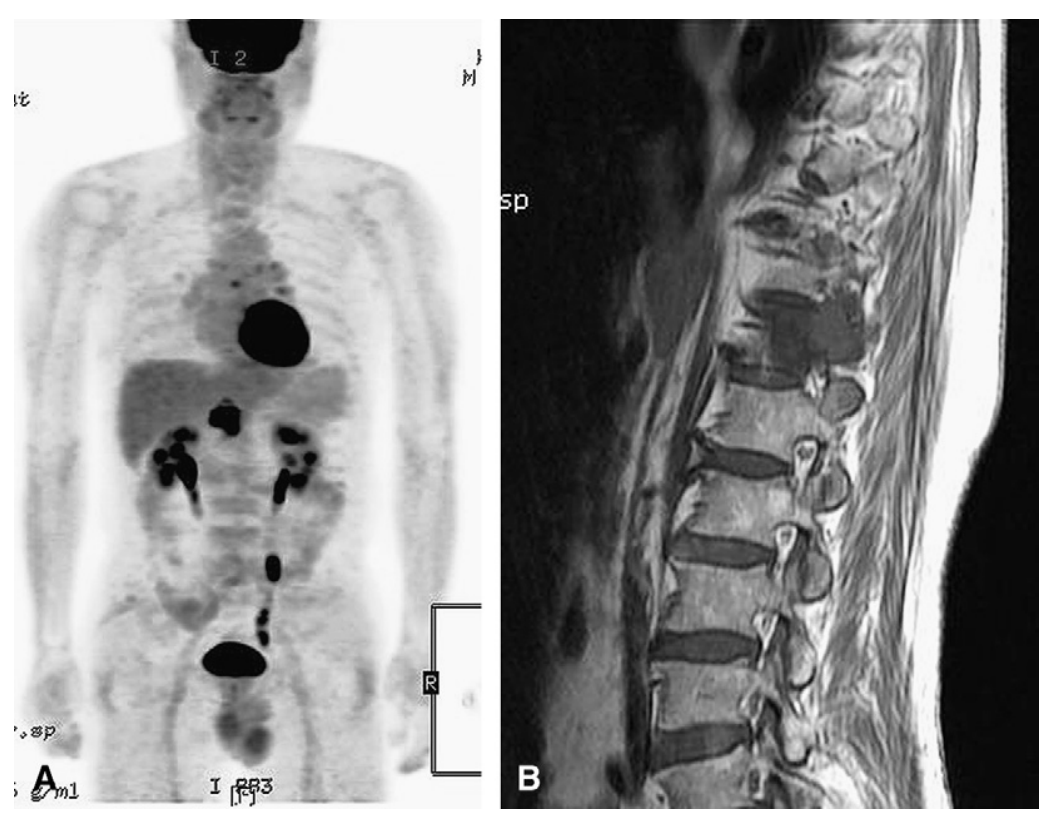

FIGURE 2. An asymptomatic patient manifested extrathoracic recurrence without intrathoracic recurrence. A, A follow-up integrated PET/CT after curative resection showed hypermetabolism at the thoracic spine. B, MRI of the spine confirmed bone metastasis of lung cancer.

abnormal findings in scheduled tests after curative resection of NSCLC. As a result, integrated PET/CT might be useful as a follow-up tool even in cases in which recurrence is not suspected after curative resection. A number of previous studies have shown the usefulness of integrated PET/CT for suspected recurrent NSCLC after curative resection. ${ }^{11-13}$ In those studies, the sensitivity, specificity, and accuracy values of integrated PET/CT for diagnosis of recurrence was around $90 \%$. As a single-time modality, therefore, integrated PET/CT can confirm suspected recurrence and be used to assess the status of disease. ${ }^{12}$ However, no studies have been conducted regarding the use of the integrated PET/CT to detect recurrence when it is not suspected after curative resection. To the best of our knowledge, the present study is the first to address integrated PET/CT in this type of clinical setting. How often and by what means surveillance should be carried out is debatable, mainly owing to the lack of evidence that earlier treatment of recurrence leads to a better outcome. ${ }^{14}$ The American Society of Clinical Oncology guidelines for NSCLC state that there is no proven value for either chest radiograph or CT in surveillance and recommend only history-taking and physical examination every 3 months. ${ }^{15}$ However, there is considerable interest in developing noninvasive, easily performed, safe, and accurate techniques for detecting recurrences or metachronous double primary lung cancer at the earliest possible time. Guidelines from the American College of Radiology, in addition to NCCN and ACCP guidelines, recommend a postresection chest $\mathrm{CT}$ at 3 months to establish a new baseline and then an annual check-up. ${ }^{16}$ However, it is still uncertain whether early detection of cancer recurrence in asymptom- atic patients positively affects prognosis. Virgo and associates $^{3}$ demonstrated no differences in survival time in an intensive surveillance group, and Younes, Gross, and Deheinzelin ${ }^{6}$ also found that intensive surveillance yielded no survival advantage and was more expensive than a symptom-based surveillance. Although the asymptomatic patients had a longer survival time after detection of recurrence, Walsh and colleagues ${ }^{4}$ believed that this reflected leadtime bias and not a true survival benefit. In contrast, Westeel, Choma, and Clement ${ }^{7}$ found good compliance with an intensive surveillance regimen in a subset of the entire group, and survival after recurrence for patients with asymptomatic recurrences was significantly better than for patients with symptomatic recurrences. Sugimura and coworkers ${ }^{2}$ showed treatment for recurrent NSCLC significantly prolonged survival and concluded that surgery should be considered. They also investigated independent prognostic factors of postrecurrence survival and demonstrated that the presence of symptoms was a poor prognostic factor. ${ }^{2}$ In our institution, an intensive surveillance program has been adopted after curative resection for NSCLC. It consists of regular visits, tumor marker evaluation every 3 months, and a chest CT every 6 months. When patients report specific symptoms at their regular visit, various types of diagnostics (eg, brain MRI, bone scan, and abdominal and pelvic CT) are performed to rule out recurrence. These procedures are time-consuming and costly if 2 or more diagnostics are needed. Therefore, a simpler and more efficient diagnostic tool to help determine systemic status safely and reliably is needed. For this reason, we performed a follow-up integrated PET/CT after curative resection in patients having NSCLC. We found 
the advantages of integrated PET/CT to be early detection of recurrence in the absence of symptoms or abnormal findings and extrathoracic recurrence in the absence of intrathoracic recurrence. When an asymptomatic patient has distant metastases accompanied by intrathoracic recurrence, distant metastases can be detected through other types of examination. However, when a patient with asymptomatic recurrence has extrathoracic metastasis only, early diagnosis is impossible because the routine test is focused only on the thorax. In this study, integrated PET/CT detected extrathoracic recurrence in a total of 6 patients with no intrathoracic recurrence. As for brain metastasis, the usefulness of a PET might be decreased because physiologic accumulation of FDG occurs in the brain. However, understanding of PET/ CT findings and whole-body PET/CT would be helpful in evaluating brain metastasis. ${ }^{17}$ Brain metastases in 3 patients who were asymptomatic were detected by integrated wholebody PET/CT, which was confirmed by brain MRI. Integrated PET/CT can also detect the extrathoracic double primary cancer, as shown by our results.

Our study has a number of limitations. First, there was no control group receiving an intensive surveillance with a method other than integrated PET/CT. Our study, however, was not intended to compare integrated PET/CT with chest CT. Because there are no data of integrated PET/CT in a surveillance program, our study provides new information about the early detection of cancer recurrence in asymptomatic patients with NSCLC. Second, we did not evaluate the extent to which early detection of recurrence through integrated PET/CT influenced the prognosis (an assessment of survival gain). Inasmuch as this study involved a small number of patients and the follow-up observation period was relatively short, further studies are necessary to address this. Third, although patients with abnormal findings on integrated PET/CT were found to have recurrence on 12-month follow-up imaging, not all the recurrences were confirmed through histologic examination. Fourth, differentiating a second double primary lung cancer from recurrence is difficult. We determined our cases to be recurrence rather than second double primary lung cancer because the recurrences were found within 2 years and with mediastinal node metastasis.

\section{CONCLUSIONS}

Integrated PET/CT can be a useful diagnostic tool to detect cancer recurrence in NSCLC patients without symptoms or abnormal findings. Further studies are required to evaluate the cost-effectiveness or survival benefit of follow-up integrated PET/CT.

\section{References}

1. Clifton F, Mountain MD. Revisions in the international system for staging lung cancer. Chest. 1997;111:1711-7.

2. Sugimura H, Nichols FC, Yang P, Allen MS, Cassivi SD, Deschamps CD, et al. Survival after recurrent non small-cell lung cancer after complete pulmonary resection. Ann Thorac Surg. 2007;83:409-18.

3. Virgo KS, McKirgan LW, Caputo MCA, Mahurin DM, Chao LC, Caputo NA, et al. Post-treatment management option for patients with lung cancer. Ann Surg. 1995;222:700-10.

4. Walsh GL, O'Connor M, Willias KM, Milas M, Wong RS, Nesbitt JC, et al. Is follow-up of lung cancer patients after resection medically indicated and costeffective? Ann Thorac Surg. 1995;60:1563-72.

5. Edelman MJ, Meyers FJ, Siegel D. The utility of follow-up testing after curative cancer therapy. A critical review and economic analysis. J Gen Intern Med. 1997; 12:318-31.

6. Younes RN, Gross JL, Deheinzelin D. Follow-up in lung cancer. How often and for what purpose? Chest. 1999;115:1494-9.

7. Westeel V, Choma D, Clement F. Relevance of an intensive postoperative followup after surgery for non-small lung cancer. Ann Thorac Surg. 2000;70:1185-90.

8. Rubins J, Unger M, Colicce GL. Follow-up and surveillance of the lung cancer patient following curative intent therapy: ACCP evidence-based clinical practice guideline (2nd edition). Chest. 2007;132:355-67.

9. National Comprehensive Cancer Network. Practice guidelines for non-small cell lung cancer. Rockledge (PA): National Comprehensive Cancer Network; 2000.

10. Bruzzi JF, Munden RF. PET/CT imaging of lung cancer. J Thorac Imaging. 2006; 21:123-36.

11. Hicks RJ, Kalff V, MacManus MP, Ware RE, McKenzie AF, Matthews JP, et al The utility of F-FDG PET for suspected recurrent non-small cell lung cancer after potentially curative therapy: impact on management and prognostic stratification. J Nucl Med. 2001;42:1605-13.

12. Keidar Z, Haim N, Guranlnik L, Wollner M, Bar-Shalom R, Ben-Nun A, et al. PET/CT using 18F-FDG in suspected lung cancer recurrence: diagnostic value and impact on patient management. J Nucl Med. 2004;45:1640-6.

13. Inoue T, Kim EE, Komaki R, Wong FC, Bassa P, Wong WH, et al. Detecting recurrent or residual lung cancer with FDG-PET. J Nucl Med. 1995;36:788-93.

14. Benamore R, Shepherd FA, Leighl N, Pintilie M, Patel M, Feld R, et al. Does intensive follow-up alter outcome in patients with advanced lung cancer? J Thorac Oncol. 2007;2:273-81.

15. American Society of Clinical Oncology treatment of unresectable non-small cell lung cancer guideline: update 2003. J Clin Oncol. 2004;22:330-53.

16. Follow-up of non-small cell lung cancer: American College of Radiology appropriateness criteria, 2005.

17. Lee HY, Chung JK, Jeong JM, Lee DS, Kim DG, Jung HW, et al. Comparison of FDG-PET findings of brain metastasis from non-small cell lung cancer and smallcell lung cancer. Ann Nucl Med. 2008;22:281-6. 